\title{
Interaction of Atoms with Grain Surfaces in Steel: Periodic Dependence of Binding Energy on Atomic Number and Influence on Wear Resistance
}

\author{
Yuri F. Migal ${ }^{1}$, Vladimir I. Kolesnikov ${ }^{2}$, Vladimir N. Doronkin ${ }^{2}$, Evgeni S. Novikov ${ }^{2}$ \\ ${ }^{1}$ Southern Scientific Centre of Russian Academy of Sciences, Rostov-on-Don, Russia \\ ${ }^{2}$ Rostov State Transport University, Rostov-on-Don, Russia \\ Email:ymigal@mail.ru
}

Received July 29, 2012; revised September 10, 2012; accepted September 21, 2012

\begin{abstract}
Quantum-chemical calculations of polyatomic clusters simulating a boundary between grains in a surface layer of steel were carried out. Along with iron atoms the clusters contain atoms of alloying and impurity elements which appear on the boundary due to grain boundary segregation or intergrain diffusion. The influence of the chemical composition of a segregate on the strength of coupling between grains and, eventually, on steel wear resistance is analyzed. Results obtained show that the degree of the strength of binding of segregated atoms with atoms of iron in a metal surface layer is an essential factor influencing wear resistance. It is found that the dependence of energy of binding of atoms of different elements with grain surface on the atomic number complies with the periodic law. This fact can be considered as a theoretical base for the prognostication of strength properties of steel with different composition of alloying and impurity elements. Potential energy curves corresponding to the movement of atoms on iron surface are studied. They can be useful for design of the composition of multi-layer coats on steel.
\end{abstract}

Keywords: Steel; Grain Boundary; Compatibility of Elements; Wear Resistance; DFT

\section{Introduction}

Wear resistance of steel and other polycrystalline materials is known to be determined, in many respects, by properties of boundaries between grains of surface layers. Although the structure of grains of these layers can essentially differ from the ones of inner domains of polycrystals, the mechanisms of destruction of both surfaces and inner domains are similar in broad terms. One of the causes of destruction of polycrystals mentioned in literature is grain boundary segregation during which atoms of alloying and impurity elements go out from the bulk of grains to their boundaries. As a result the bond between grains can be weakened. This idea was stated long ago, but in spite of numerous experimental studies of the grain boundary segregation, now the connection between this phenomenon and the mechanism of destruction of polycrystalline materials has not yet been completely clarified.

There are different points of view how segregated atoms contribute to destruction (see, e.g., [1-5]). One of the hypotheses is based on the fact that in process of grain boundary segregation the metal-metal bond is substituted by a weaker or stronger bond of metal with segregated atoms [1]. Confirming this hypothesis, the estimations of energy amount required to break the bond between grains were performed in [6]. It should be noted that here only the data of the sublimation enthalpy and sizes of atoms of the substances to be studied were used. It is striking that results of these estimations, in spite of the very simplified approach, are in accord, in whole, with known experimental data relating to embrittlement of steel. This fact gives a reason to suppose that the magnitude of the binding energy of atoms in many respects determines the ability of segregated atoms to weaken or strengthen grain boundaries. Nevertheless, in view of the assumptions forming the basis of the estimations, this conclusion needs experimental substantiation and/or additional research.

Quantum chemistry methods based on the approximation of the density functional theory (DFT) appearing in recent decades allow for more accurate analysis (than in [6]) of atomic interactions affecting metal destruction.

These methods have turned out to be effective for investigating various phenomena wherein chemical interactions can be observed. However, the researches, in which these and similar quantum-chemical methods are applied to revealing the connection between the phenomenon of grain boundary segregation and the problem 
of destruction and wear resistance of metals, are still rare, and segregation only of some selected elements is considered in them (see, e.g., [3-5] where the segregation of the H, B, C, S, P, Mn and some other elements was studied). In our opinion, for understanding the problem more deeply, it is necessary to perform a more systematic analysis for studying a large group of segregated elements.

In our computations concerning segregation, we used the ADF software [7] based on the DFT approximation. It makes possible the study of the compounds of transitional metals including hundreds of atoms. That is important for applications in case of steel. The interactions between grains in presence of segregated atoms were studied by means of model polyatomic clusters. This analysis allowed us to make sure that the appearance of segregated atoms on grain boundaries is not an attendant effect, but one of the main causes of weakening the strength of the bond between grains and the following destruction of a surface layer of metal [8].

In what follows we also show that the quantumchemical approach allows us to perform the systematization of atomic interactions on grain-boundary surfaces, estimate the compatibility of different elements with iron on these surfaces, and connect this compatibility with the Mendeleyev's periodic law. Our calculations of potential energy curves for atoms of chemical elements, situated on outer or grain surfaces of steel, make possible to perform a comparative analysis of mechanical stress appearing in transitional layers between iron and other elements. In our opinion, these results could be useful for the development of microscopic theory of multi-layer coats of metals.

\section{Choice of Cluster Model}

It is obvious that the whole quantum-chemical analysis of such an inhomogeneous macroscopic system as polycrystal, is practically not possible because of a huge number of atomic interactions in the system. In this case it is appropriate to select some microscopic part (a polyatomic cluster) from a polycrystal, in which the phenomenon in question can be studied in detail. As an alternative, one can use so-called zone approach, within which a grain-boundary surface is accepted to be infinite. However, within this approach it is practically impossible to estimate the energy of a chemical bond between interacting fragments. That is why the cluster model was chosen.

A polyatomic cluster imitating the segregation in a polycrystalline material should evidently comprise some amount of matrix atoms (from the adjacent layers of two neighboring grains) as well as segregated or diffused atoms located on the boundary. The cluster size should be large enough to more precisely reproduce basic quali- tative and quantitative regularities of the segregation. However, the practice of quantum-chemical calculations shows that in many cases rather small clusters can reproduce features of the phenomenon studied on qualitative or semi-quantitative levels (see, e.g., [9]).

We have selected the binding energy $E_{b}$ of a cluster and interatomic distances as calculated characteristics. The energy $E_{b}$, obtained through the ADF package and taken modulo, approximately represents the energy necessary for the breaking of all bonds in a cluster. As a cluster size increases, the binding energy $E_{b}(1)$ per atom should modulo monotonically increases, and theinteratomic distances should approach values specific for a crystal lattice.

The results of calculations of dependence of $E_{b}(1)$ on the number $n$ of atoms in a cluster are represented in Figure 1. From this figure it follows, that as $n$ value changes from 2 up to 8 the binding energy grows rather quickly. But when $n=18$ the dependence $E_{b}(1)$ on $n$ is stabilized. Basing on this result, we suppose that a cluster with $n=18$ can be considered as the minimal cluster which can be used for investigating the phenomena related to variations of the element composition in steel. In particular, we expect that the usage of clusters of a similar size allows us to study variations of the binding energy caused by the grain boundary segregation. This conclusion is preliminary, considering that the features of the grain boundary segregation were not taking in above estimations. The final conclusion concerning the sizes of clusters suitable for study of this phenomenon will be made below, after the comparison of obtained results with known data.

In simulation of the conditions under which segregation occurs, we used a well-known fact that on the narrowest portion of the boundary between grains in a polycrystal (that is, in the place of grain contact), the width of the boundary is on the order of a lattice constant. This allows us to consider the simple model of a boundary whereby the atoms of the middle layer are replaced with

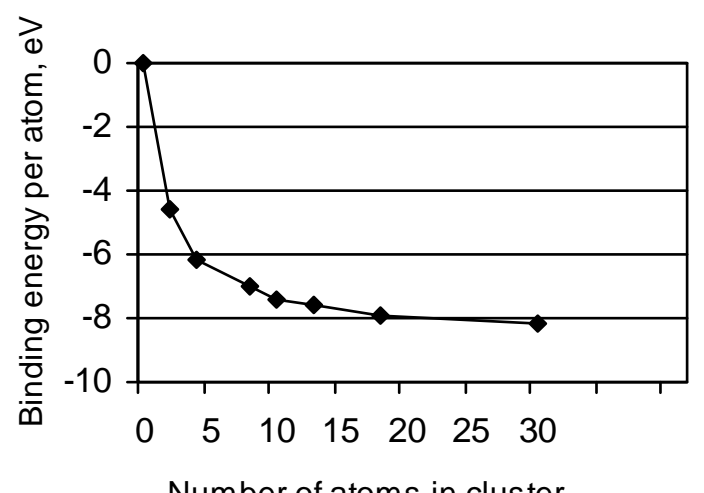

Figure 1. Dependence of the binding energy per atom on the number of atoms in a cluster. 
segregated atoms in an 18-atomic iron cluster (Figure 2). Two upper and two lower layers simulate the upper and lower grains of the polycrystal respectively, and the middle layer corresponds to the boundary between the grains. In case of high purity metal the model cluster contains only Fe atoms.

Of course, such a model does not take into account the variety of conditions that are significant for segregation and wear resistance (including, e.g., crystallite surface irregularities, the nonparallel nature of surfaces, dislocations, etc.). The main purpose of the model proposed is, firstly, to evaluate the effect of the closest atomic surrounding on the chemical bond between segregated atoms and matrix atoms in the place of the grain boundary, and, secondly, to determine how the bond affects the strength of microscopic domains and surface layers of polycrystals.

If one removes all atoms of the two upper layers in this model, then the remaining atoms (the lower layers + the layer of impurity atoms) imitate the phenomenon of adsorption on the (100) surface of iron. The results of calculations relating to two similar phenomena, the segregation of elements on a grain boundary and the adsorption on a free surface of iron, are represented in the next section. We suppose that the comparison of these results helps us to explain why the conclusions obtained in [6] by using the sublimation data (the phenomenon typical for outer surfaces) are valid for the phenomenon of grain boundary segregation typical for inner surfaces in polycrystals.

\section{Calculations of Binding Energy of Clusters Containing Adsorbed and Segregated Atoms}

We performed calculations of binding energy $E_{b}$ of clusters which contain adsorbed and segregated atoms of chemical elements with atomic numbers from 1 to 36 (from hydrogen to krypton). These additional atoms were arranged either on an outer surface of a cluster (in case of

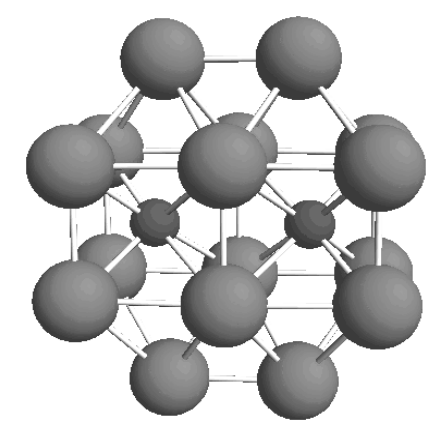

Figure 2. The minimum cluster allowing us to reproduce semi-quantitatively the interaction of segregated atoms with a steel grain surface. Big particles correspond to Fe atoms, and small to segregated atoms. adsorption) or in a middle layer (in case of segregation). In both cases the position of additional atoms was over the centre of quadrates from iron atoms (the fourfold position) or over sides of these quadrates (the twofold position). As a rule, in calculations the TZ2P basis (see [7]) and the option "Small" (frozen core orbitals) were used.

Comparing $E_{b}$ values for clusters containing adsorbed atoms and clusters consisting only of iron atoms it is possible to estimate the strength of bond of different atoms with iron. If adsorbed atoms are arranged over the centre of quadrates (i.e. in positions which are similar to those of iron atoms in a matrix), it can be accepted formally that the adsorbed atoms substitute iron atoms in a cluster of a greater size. In such cases it is easy to determine which bond is stronger: Fe-Fe or Fe-X (the symbol $\mathrm{X}$ is used to denote both adsorbed and segregated atoms). Obviously, the stronger bond corresponds to a cluster with a greater modulo binding energy.

Values $E_{b}$ for clusters with adsorbed atoms are represented in Table 1. From the table it follows that atoms of carbon, nitrogen, vanadium, chrome and manganese are more strongly connected with an iron surface, than iron atoms with each other. On the other hand, atoms of lithium, sulfur, aluminum and copper are more weakly connected with the surface.

The results of calculations for clusters containing segregated atoms are represented in Table 2. As follows from the data, carbon, nitrogen, chrome and manganese atoms sew together strongly the iron grains since the binding energy of the clusters containing these atoms appears to be lower than the binding energy of the samesized cluster consisting of pure iron. Conversely, the atoms of lithium, silicon, phosphorus, sulfur, copper, and nickel, though entering into chemical bonds with iron, weaken the bond between the grains since the binding energy of the corresponding clusters is less than the binding energy of the pure iron cluster. Evidently, the weakening of the intergrain bond in the surface layer decreases steel wear resistance.

Table 1. Binding energy of adsorptive clusters $\mathrm{Fe}_{2} \mathrm{Fe}_{6} \mathrm{X}_{2}$ (eV).

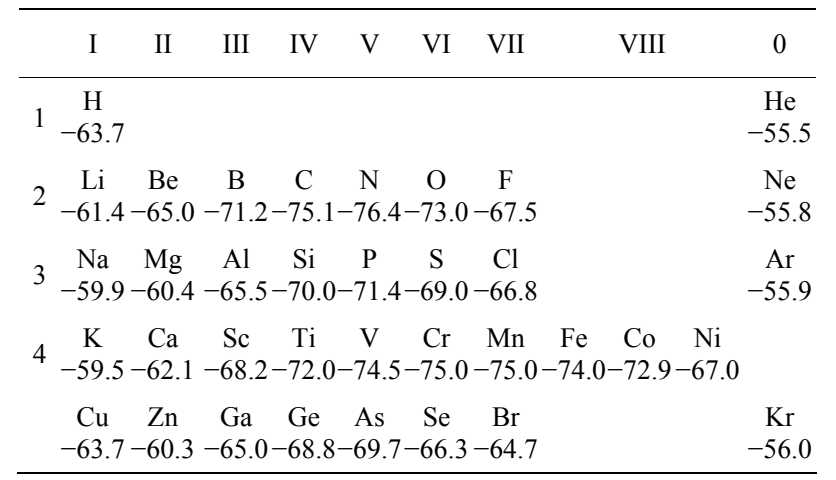


Table 2. Binding energy of segregate clusters $\mathrm{Fe}_{2} \mathrm{Fe}_{6} \mathrm{X}_{2} \mathrm{Fe}_{2} \mathrm{Fe}_{6}(\mathrm{eV})$.

\begin{tabular}{|c|c|c|c|c|c|c|c|c|c|c|c|}
\hline & I & II & III & IV & V & VI & VII & \multicolumn{3}{|c|}{ VIII } & 0 \\
\hline 1 & $\begin{array}{c}\mathrm{H} \\
-129.4\end{array}$ & & & & & & & & & & $\begin{array}{c}\mathrm{He} \\
-113.0\end{array}$ \\
\hline 2 & $\begin{array}{c}\mathrm{Li} \\
-120.6\end{array}$ & $\begin{array}{c}\mathrm{Be} \\
-127.6\end{array}$ & $\begin{array}{c}\text { B } \\
-138.7\end{array}$ & $\begin{array}{c}\mathrm{C} \\
-143.2\end{array}$ & $\begin{array}{c}\mathrm{N} \\
-140.9\end{array}$ & $\begin{array}{c}\mathrm{O} \\
-136.5\end{array}$ & $\begin{array}{c}\mathrm{F} \\
-125.9\end{array}$ & & & & $\begin{array}{c}\mathrm{Ne} \\
-112.5\end{array}$ \\
\hline 3 & $\begin{array}{c}\mathrm{Na} \\
-117.7\end{array}$ & $\begin{array}{c}\mathrm{Mg} \\
-120.8\end{array}$ & $\begin{array}{c}\mathrm{Al} \\
-127.6\end{array}$ & $\begin{array}{c}\mathrm{Si} \\
-133.3\end{array}$ & $\begin{array}{c}\mathrm{P} \\
-134.9\end{array}$ & $\begin{array}{c}\mathrm{S} \\
-131.2\end{array}$ & $\begin{array}{c}\mathrm{Cl} \\
-124.1\end{array}$ & & & & $\begin{array}{c}\mathrm{Ar} \\
-112.4\end{array}$ \\
\hline 4 & $\begin{array}{c}\mathrm{K} \\
-116.9\end{array}$ & $\begin{array}{c}\mathrm{Ca} \\
-121.8\end{array}$ & $\begin{array}{c}\mathrm{Sc} \\
-129.8\end{array}$ & $\begin{array}{c}\mathrm{Ti} \\
-135.9\end{array}$ & $\begin{array}{c}\mathrm{V} \\
-140.0\end{array}$ & $\begin{array}{c}\mathrm{Cr} \\
-141.7\end{array}$ & $\begin{array}{c}\mathrm{Mn} \\
-141.9\end{array}$ & $\begin{array}{c}\mathrm{Fe} \\
-140.2\end{array}$ & $\begin{array}{c}\text { Co } \\
-137.7\end{array}$ & $\begin{array}{c}\mathrm{Ni} \\
-130.9\end{array}$ & \\
\hline & $\begin{array}{c}\mathrm{Cu} \\
-125.7\end{array}$ & $\begin{array}{c}\mathrm{Zn} \\
-121.4\end{array}$ & $\begin{array}{c}\mathrm{Ga} \\
-126.6\end{array}$ & $\begin{array}{c}\mathrm{Ge} \\
-130.3\end{array}$ & $\begin{array}{c}\text { As } \\
-130.9\end{array}$ & $\begin{array}{c}\mathrm{Se} \\
-125.9\end{array}$ & $\begin{array}{c}\mathrm{Br} \\
-121.2\end{array}$ & & & & $\begin{array}{c}\mathrm{Kr} \\
-112.1\end{array}$ \\
\hline
\end{tabular}

Reasoning about correspondence between the binding energy of a cluster and the strength of grain bonds is confirmed by calculations of energy $E_{d}$ which is necessary for dividing a cluster into two parts imitating grains in a model cluster. Values of this quantity can be determined by the simple equation

$$
E_{d}=\left|E_{s}-E_{g}-E_{a}\right|,
$$

where $E_{s}$ is the binding energy of a segregate cluster including two grains and a boundary layer between them, $E_{g}$ is the binding energy of a cluster consisting of only one grain, and $E_{a}$ is the binding energy of an adsorptive cluster consisting of a grain and a boundary layer. By using the same equation it is possible to calculate the energy of disjoining a cluster from pure iron, supposing that the 18-atomic cluster $\mathrm{Fe}_{2} \mathrm{Fe}_{6} \mathrm{Fe}_{2} \mathrm{Fe}_{6} \mathrm{Fe}_{2}$ can be divided into two parts $\mathrm{Fe}_{2} \mathrm{Fe}_{6} \mathrm{Fe}_{2}$ and $\mathrm{Fe}_{6} \mathrm{Fe}_{2}$.

In this way we can estimate the energy of bond of iron grains separated by atoms of different elements. The results of such calculations are given in Table 3 . This table, depicted by analogy with the Mendeleyev's periodic table, actually reflects the compatibility of different elements with iron on its grain surfaces. For elements from the beginning of a period the bond with iron is weak comparatively with the Fe-Fe bond, and, as the atomic number grows, the bond increases at first, and then for the second half of a period it decreases. The bond becomes weaker while moving along a group. It signifies that properties of the chemical bond of different elements with iron on inner surfaces are in periodic dependence on the nuclear charge of atoms, and this corresponds to the periodic law. Table 3 shows that elements whose bond with iron is the weakest are alkaline and alkaline-earth metals, and also inert gases. These elements mostly soften steel. Besides, softening elements are those from the middle of the third period ( $\mathrm{Si}, \mathrm{P}, \mathrm{S})$. As it is generally known, phosphorus and sulfur entering into steel deteriorate its properties. As we can see, this is conditioned by the small values of the energy of binding these elements with iron.
Table 3. Energy necessary for dividing segregate clusters $\mathrm{Fe}_{2} \mathrm{Fe}_{6} \mathrm{X}_{2} \mathrm{Fe}_{2} \mathrm{Fe}_{6}(\mathrm{eV})$.

\begin{tabular}{|c|c|c|c|c|c|c|c|c|c|c|c|}
\hline & I & II & III & IV & V & VI & VII & \multicolumn{3}{|c|}{ VIII } & 0 \\
\hline 1 & $\begin{array}{c}\mathrm{H} \\
9.9\end{array}$ & & & & & & & & & & $\begin{array}{l}\mathrm{He} \\
1.7\end{array}$ \\
\hline 2 & $\begin{array}{r}\mathrm{Li} \\
3.4\end{array}$ & $\begin{array}{l}\mathrm{Be} \\
6.8\end{array}$ & $\begin{array}{c}\text { B } \\
11.7\end{array}$ & $\begin{array}{c}\mathrm{C} \\
12.3\end{array}$ & $\begin{array}{c}\mathrm{N} \\
8.7\end{array}$ & $\begin{array}{c}\mathrm{O} \\
7.8\end{array}$ & $\begin{array}{c}F \\
2.7\end{array}$ & & & & $\begin{array}{l}\mathrm{Ne} \\
1.0\end{array}$ \\
\hline 3 & $\begin{array}{l}\mathrm{Na} \\
2.0\end{array}$ & $\begin{array}{l}\mathrm{Mg} \\
4.6\end{array}$ & $\begin{array}{l}\mathrm{Al} \\
6.3\end{array}$ & $\begin{array}{r}\mathrm{Si} \\
7.5\end{array}$ & $\begin{array}{c}\mathrm{P} \\
7.7\end{array}$ & $\begin{array}{c}S \\
6.4\end{array}$ & $\begin{array}{c}\mathrm{Cl} \\
1.6\end{array}$ & & & & $\begin{array}{l}\mathrm{Ar} \\
0.7\end{array}$ \\
\hline 4 & $\begin{array}{c}\mathrm{K} \\
1.6\end{array}$ & $\begin{array}{l}\mathrm{Ca} \\
3.9\end{array}$ & $\begin{array}{c}\mathrm{Sc} \\
5.8\end{array}$ & $\begin{array}{r}\mathrm{Ti} \\
8.1\end{array}$ & $\begin{array}{l}\mathrm{V} \\
9.7\end{array}$ & $\begin{array}{c}\mathrm{Cr} \\
10.9\end{array}$ & $\begin{array}{c}\mathrm{Mn} \\
11.1\end{array}$ & $\begin{array}{c}\mathrm{Fe} \\
10.4\end{array}$ & $\begin{array}{l}\text { Co } \\
9.0\end{array}$ & $\begin{array}{l}\mathrm{Ni} \\
8.1\end{array}$ & \\
\hline & $\begin{array}{l}\mathrm{Cu} \\
6.2\end{array}$ & $\begin{array}{l}\mathrm{Zn} \\
5.3\end{array}$ & $\begin{array}{l}\mathrm{Ga} \\
5.8\end{array}$ & $\begin{array}{l}\mathrm{Ge} \\
5.8\end{array}$ & $\begin{array}{l}\text { As } \\
5.4\end{array}$ & $\begin{array}{c}\mathrm{Se} \\
3.8\end{array}$ & $\begin{array}{c}\mathrm{Br} \\
0.8\end{array}$ & & & & $\begin{array}{l}\mathrm{Kr} \\
0.3\end{array}$ \\
\hline
\end{tabular}

Comparing the characteristics of atoms in adsorbed and segregated states, it is possible to conclude that atoms weakly bonded with a free iron surface also weakly interact with iron on a grain boundary. Evidently, it is possible to suppose, that the bond strength depends more on the type of atoms tied together, than on the number and location of atoms of the nearest environment. Although, undoubtedly, the dependence of bond on the nearest environment exists, on a qualitative level, when the influence of different elements on wear resistance is studied, this fact can be ignored. Besides, we are convinced that the bond strength is a rather stable quantity with respect to methods of the estimation. It is confirmed, in particular, by the fact that the results of our calculations qualitatively agree with results in [6], in spite of significant difference in the approaches.

The stated above explains why the clusters of rather small sizes used by us allow the modeling of changes of the strength of grain bonds in the grain boundary segregation process.

Taking into account all these facts, it is possible to formulate the following important conclusion: the elements, whose energy of binding with iron is much less than the energy of the Fe-Fe bond, reduce the wear resistance of steel. If these atoms appear on a boundary between two grains in a surface layer of a polycrystal, their weak bond with iron is the essential factor influencing 
the strength of this layer.

In spite of an approximate character of our calculations, the results obtained conform with known experimental facts and correspond to the periodic law. We suppose that more precise calculations will confirm the obtained dependence of hardening (or softening) properties of elements on the nuclear charge of atoms. This dependence can be a theoretical base for the prediction of strength properties of polycrystalline materials with different alloying components.

When introducing hardening components into steel it should be taken into account that the entered elements can dissolve in a volumetric phase of iron (i.e. within grains) and a part of them, which remains on surfaces of grains, can be too small and insufficient for steel hardening. From this point of view the alloying of steel by boron appears to be most effective, because boron having a smaller value of $E_{d}$ than carbon, is dissolved in steel much less than carbon. If steel is alloyed by boron in amounts providing the filling of grain boundaries (actually it is the 100-th fractions of percent of the total volume of a sample), it is possible to expect an essential increase of steel wear resistance. However, if more boron is added, some boron atoms will penetrate into iron grains, and there will appear compounds of boron with iron, carbon and other metals inside them-borides and carbides. These compounds are considerably less strong than iron, and properties of steel will be deteriorated. Thus, it is possible to raise the strength of steel only by adding small concentrations of boron.

\section{Potential Energy Curves for Adsorbed and Segregated Atoms}

The adduced above values of the binding energy of clusters, in fact, correspond to the separation of grains in a direction perpendicular to a grain boundary surface. In this case no work to overcome friction forces between grains is performed. Under real conditions, at least some part of grains moves with a shift relatively to each other during destruction of a polycrystalline material. To take into account the additional losses of energy appearing in this case it is necessary to consider a relief of potential energy for impurity atoms moving along a grain boundary surface.

To calculate this quantity for a model cluster, we supposed that coordinates of all Fe atoms are fixed and atoms of investigated elements may be displaced along and perpendicularly to a grain surface (Figure 3 ). The trajectory of an atom should correspond to minimal values of energy as the atom moves from one equilibrium position to another.

Figure 4 shows the calculated curves describing the relief of the potential energy $E(x)$, where $x$ is the coor-

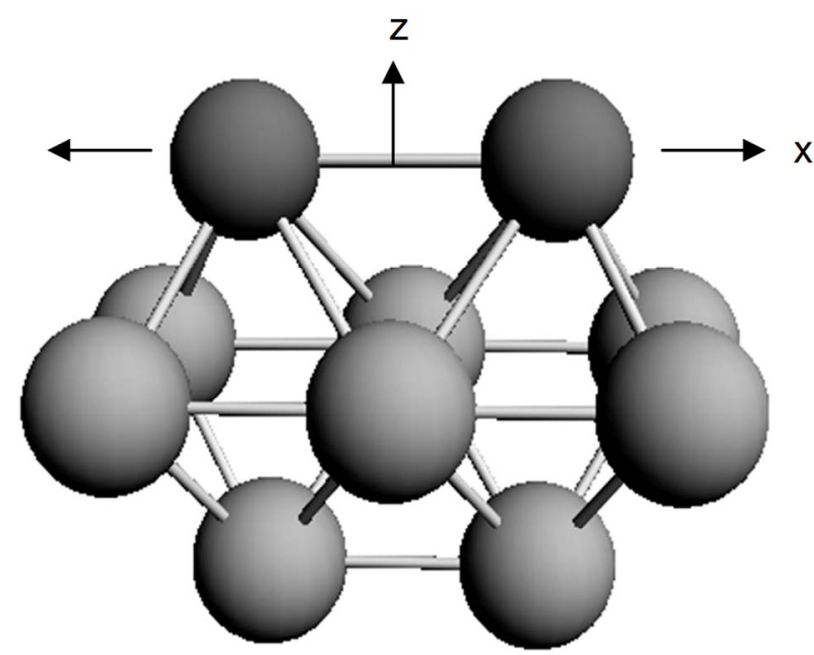

Figure 3. Coordinates describing the displacement of atoms on iron surface.

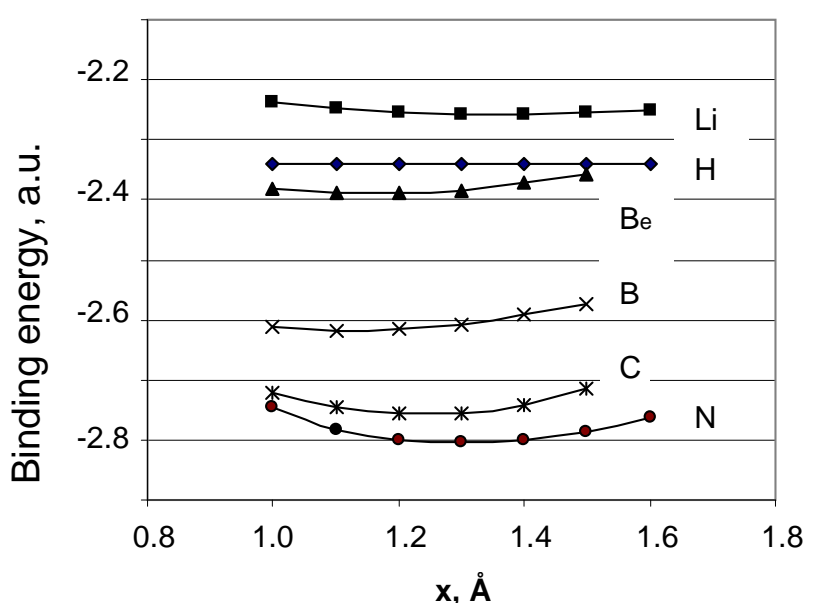

Figure 4. Relief of the potential energy for elements of the first and second periods.

dinate of the atom on the surface, for adsorbed atoms of some elements. We can see that, really, the steepness of the relief for various elements can differ noticeably. The relief for hydrogen is most flat. The energy of its interaction with an iron surface practically does not depend on a position of hydrogen atom on the surface. Evidently, it corresponds to the known fact that hydrogen does not create any steady compounds with iron. For elements of the second period the steepness of the relief increases as the atomic number of an element grows.

An important quantitative characteristic of the relief of the potential energy $E(x)$ is the second derivative $d^{2} E / d x^{2}$ at the equilibrium position. The more this derivative is, the steeper is the relief of $E(x)$ and the more difficult it is to displace the atom from the equilibrium position. The values of this derivative for the case of adsorbed and segregated atoms are represented in Figure 5. 


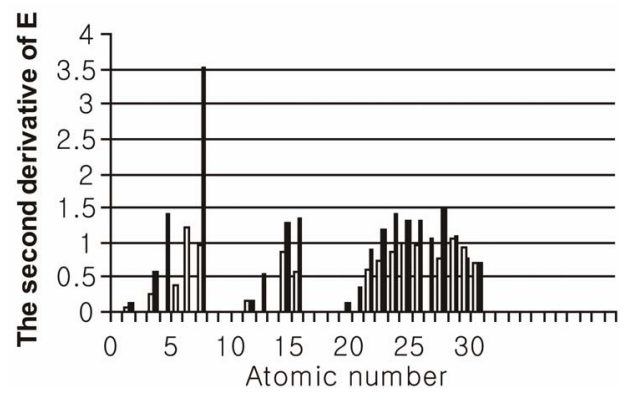

Figure 5. Values of the second derivative $d^{2} E / d x^{2} \quad$ (a.u. $/ \AA^{2}$ ) for elements adsorbed (light columns) and segregated (dark columns) on iron surface.

This figure shows that the steepness of the relief for elements from the beginning and the end of a period is comparatively small, and is greater for elements from the middle of the period. This fact correlates with the behavior of energy of the binding of atoms with a surface studied above. Namely, atoms of elements, which are separable with difficulty from an iron surface in the perpendicular direction, also cannot be freely moved in parallel directions. It signifies, in particular, that if a certain element has a property to strengthen the bond between iron grains, this property does not depend on the direction of a destroying action.

The degree of steepness of the relief of the potential energy of adsorbed or segregated atoms can be an essential factor influencing the stability of a layer containing these atoms. When the size of the atoms exceeds the size of iron atom, the order and symmetry in disposition of atoms in a layer will be lower, than in the iron lattice. It can lead to a rise of energy of the layer. Since the part of atoms will be not in positions with minimal energy, so the steeper is the relief, the larger store of energy has the layer. Such an increase of energy results in appearing additional mechanical stresses in the layer. An insignificant thermal or mechanical action can lead to the essential rebuilding of the layer structure.

Practical consequence of the mentioned above is the necessity of taking into account the steepness of the relief of the potential energy for designing multi-layer coats. From general reasons it follows, that in such coats it is expedient to use the first layer from "soft" materials, i.e. such materials whose atoms have a relatively flat relief of the potential energy on a surface of the base metal. The relief is flat enough for elements of some transition metals represented in Figure 5 (copper, titanium, vanadium). Probably, this explains the fact that these metals are widely used for intermediate coats on steel.

\section{Summary}

The results of quantum-chemical calculations performed show the following.

1) Using polyatomic clusters with the number of atoms equal to 18 or more allows us to study the changes of energy of the bond between segregated atoms and surfaces of iron grains on qualitative and semi-quantitative levels. This fact indicates that the bond of atoms of various elements with iron surface is the strong effect which is well reproduced by model clusters with a rather small number of interacting atoms.

2) The comparison of the binding energy of clusters, representing adsorptive and segregate complexes of onetype elements, points to the correlation between energy of the $\mathrm{Fe}-\mathrm{X}$ bond appearing at the adsorption of an element $\mathrm{X}$ on iron surface, and energy of the $\mathrm{Fe}-\mathrm{X}-\mathrm{Fe}$ bond appearing at the grain boundary segregation.

3 ) If the energy of the Fe-X bond is greater modulo than the energy of the Fe-Fe bond, the element $\mathrm{X}$ increases steel wear resistance in the segregation process. Thus, the strength of the bond of segregated atoms with iron atoms within a surface layer of metal is an essential factor influencing wear resistance.

4) If a certain element can strengthen the bond between iron grains, this property does not depend on the direction of a destroying action.

5) The calculations have revealed the periodic dependence of energy of binding of atoms of different elements with a grain surface on their atomic number. This fact complies with the periodic law and can be considered as a theoretical base for the prognostication of strength properties of steel with different composition of alloying and impurity elements.

6) Many-layer coats of metals should have the first layer from materials whose atoms have a flat relief of the potential energy on a surface of the base metal.

\section{Acknowledgements}

The authors would like to thank Russian Foundation for Basic Research (project No. 11-08-00640-a) for supporting our work.

\section{REFERENCES}

[1] D. Briggs and M. P. Seah, "Practical Surface Analysis by Auger and X-Ray Photoelectron Spectroscopy," John Wiley \& Sons, Ltd., Chichester, 1990.

[2] G. S. Painter and F. W. Averill, "Effects of Segregation on Grain-Boundary Cohesion: A Density-Functional Cluster Model of Boron and Sulfur in Nickel," Physical Review Letters, Vol. 58, No. 3, 1987, pp. 234-237. doi:10.1103/PhysRevLett.58.234

[3] R. Yang, R. Z. Huang, Y. M. Wang, H. Q. Ye and C. Y. Wang, "The Effects of 3d Alloying Elements on Grain Boundary Cohesion in Gamma-Iron: A First Principles Study on Interface Embrittlement Due to the Segregation," Journal of Physics: Condensed Matter, Vol. 15, No. 
49, 2003, pp. 8339-8349.

[4] J. S. Braithwaite and R. Peter, "Grain Boundary Impurities in Iron," Acta Materialia, Vol. 53, No. 9, 2005, pp. 2715-2726. doi:10.1016/j.actamat.2005.02.033

[5] S. B. Gesari, M. E. Pronsato and A. Juan, "Effect of Manganese on Grain Boundary Segregation of Sulfur in Iron," Applied Surface Science, Vol. 253, No.14, 2007, pp. 5939-5942. doi:10.1016/j.apsusc.2006.12.113

[6] M. P. Seah, "Adsorption-Induced Interface Decohesion," Acta Metallurgica, Vol. 28, No. 7, 1980, pp. 955-962. doi:10.1016/0001-6160(80)90112-1

[7] G. te Velde, F. M. Bickelhaupt, S. J. A. van Gisbergen, C. Fonseca Guerra, E. J. Baerends, J. G. Snijders and T. Ziegler, "Chemistry with ADF," Journal of Computa- tional Chemistry, Vol. 22, No. 9, 2001, pp. 931-967. doi:10.1002/jcc.1056

[8] V. I. Kolesnikov, A. T. Kozakov and Yu. F. Migal, "Study of Friction and Wear in the Wheel-Rail System by X-Ray Electron and Auger-Electron Spectroscopy and Quantum Chemistry," Journal of Friction and Wear, Vol. 31, No. 1, 2010, pp. 11-22. doi: $10.3103 / \mathrm{S} 1068366610010022$

[9] M. V. Lebedev, "Mechanism of $\mathrm{H}_{2} \mathrm{~S}$ Molecule Adsorption on the GaAs(100) Surface: Ab Initio Quantum-Chemical Analysis," Physics of the Solid State, Vol. 48, No. 1, 2006, pp. 164-171. 